\section{Response to lubiprostone in chronic constipation is associated with increased mucus and mucin output: a randomized clinical trial}

\section{To the Editor,}

A prostaglandin E1 derivative, lubiprostone, is an effective treatment for chronic constipation (CC), increasing fluid secretion and lubrication of the intestinal lumen.

Lubiprostone is a chloride channel ClC-2 activator [1, 2 ], generating outflow of chloride into the lumen of the gastrointestinal tract and stimulating intestinal mucus secretion [1, 3-5].

We studied the effect of lubiprostone on gastric mucus and mucin output in chronic constipated patients, recruited at the Kansas University Medical Center, Kansas City, KS (IRB approval number 07-022L, October 2002). All subjects signed the informed consents.

Our double-blind, randomized, placebo-controlled study included 20 patients with symptoms of chronic idiopathic constipation (CIC) diagnosed according to the Rome III criteria and 20 healthy controls. All CIC patients and controls were randomized to a one-week therapy with lubiprostone at a dose of $24 \mu \mathrm{g}$ two times daily or a placebo followed by a one-week washout period, to let patients stay drug-free before switching to the next medication after which a crossover of the medication was made. Gastric juice was collected basally and during stimulation with pentagastrin $(6 \mu \mathrm{g} / \mathrm{kg}$ body weight subcutaneously) at the end of both weeks of administered treatment. The mucus content in gastric juice was assessed gravimetrically. The mucin content was determined after its purification using ultracentrifugation. The viscosity of the gastric secretion was estimated using a digital viscometer [3]. A block randomization method was used with each block consisting of five patients. Data are presented as mean \pm standard error of mean (SEM) or median (interquartile range, for Bristol Stool Scale). Independent t-test (for age) and two-way analysis of variance followed by Tukey's post hoc or Wilcoxon-rank test (for Bristol Stool Scale) were used for statistical comparisons.

The stool frequency and Bristol Stool Scale were significantly lower in CIC patients (by 52.2\%) compared to healthy controls. Moreover, a trend to lower basal mucus (by 37.5\%), mucin I (by $36.4 \%$ ), mucin II (by 29.4\%), and mucin III (by $37.1 \%$ ) outputs were found in CIC patients compared with controls, without statistical significance. Stimulation with pentagastrin significantly increased mucus output and mucin II and III outputs in the CIC patients. In healthy controls, lubiprostone did not change the bowel frequency, Bristol Stool Scale and mucus as well as the mucin output parameters compared to a placebo. In CIC patients, lubiprostone significantly increased the stool frequency, Bristol Stool Scale and basal mucus output (Fig. 1A-B). Lubiprostone did not affect pentagastrinstimulated mucus output (Fig. 1C) in CIC patients. As regards mucin subtypes, basal mucin I, II and III were higher in lubiprostone-treated CIC patients compared to placebo (Fig. 1D-F)

Symptomatic response to lubiprostone was observed in 12 (60 \%) CIC patients; there was a higher stool frequency and Bristol Stool Scale after treatment with lubiprostone (by $50.1 \%$ ) in responders compared to a placebo. In responders, basal mucus output tended to be higher after lubiprostone and basal mucin I, II and III were bordered on being statistically significant $(\mathrm{p}=0.08)$ compared to a placebo. A significantly higher content of gastric mucus and mucin in non-stimulated samples from patients with CIC after treatment with lubiprostone suggests this might be the main effect of lubiprostone. Consequently, increased viscosity might promote and/or stimulate the transportation and removal of non-digestible food components. Although increase in mucus and mucin were also noticed in stimulated conditions, neither was statistically significant. Based on our study, we hypothesize that, in CIC patients, the overall evident increase of mucus and its major component, mucin secretion, during lubiprostone administration may partially elucidate its clinical efficacy.

Importantly, not all patients with CIC responded in terms of stool frequency and Bristol Stool Scale to lubiprostone 

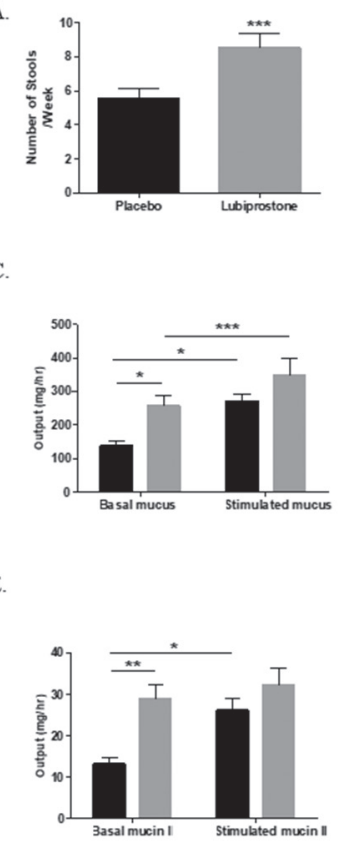

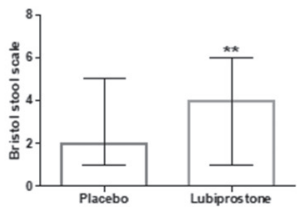

D.
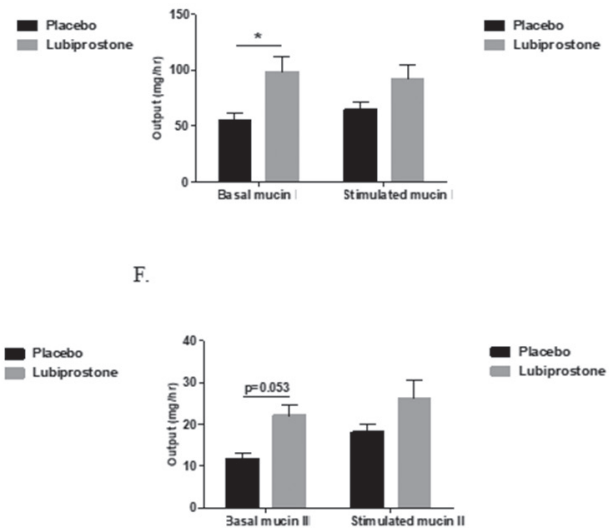

Fig. 1. The effect of administration of lubiprostone or placebo in patients with constipation.

administration. Responders had a significant increase in the number of bowel movements per week and in their basal mucin I, II, and III output, in contrast to non-responders.

Our data suggest that measuring mucin output after lubiprostone administration may predict response. Moreover, applying the minimum dose to all patients followed by dose growth in non-responders may decline the chance of developing significant side effects. Still, further understanding of the underlying factors of this drug effect is needed, as it may be critical for the improvement of the treatment strategies.

Aleksandra Tarasiuk ${ }^{1}$, Mena Milad ${ }^{2}$, Syed T. Ahmed ${ }^{3}$, Marek Majewski ${ }^{4}$, Grzegorz Wallner ${ }^{4}$, Jakub Fichna ${ }^{1}$, Mohammad Bashashati $^{2}$, Jerzy Sarosiek ${ }^{2}$

1) Department of Biochemistry, Medical University of Lodz, Lodz, Poland; 2) Division of Gastroenterology, Department of Medicine, Texas Tech University Health Sciences Center, El Paso, TX, USA; 3) Internal Medicine, John Peter Smith Hospital, Fort Worth, TX, USA; 4) 2nd Department of General Surgery, Medical University of Lublin, Lublin, Poland

Correspondence: Jerzy Sarosiek, Jerzy.Sarosiek@ttuhsc.edu

Conflicts of interest: None.

DOI: $10.15403 /$ jgld-421

\section{REFERENCES}

1. Lacy BE, Levy LC. Lubiprostone: a chloride channel activator. J Clin Gastroenterol 2007;41:345-351. doi:10.1097/01.mcg.0000225665.68920. df

2. Johnson DA. Treating chronic constipation: How should we interpret the recommendations? Clin Drug Investig 2006;26:547-557. doi:10.2165/00044011-200626100-00001
3. Majewski M, Sarosiek I, Wallner G, Edlavitch SA, Sarosiek J. Stimulation of Mucin, Mucus, and Viscosity during Lubiprostone in Patients with Chronic Constipation may Potentially Lead to Increase of Lubrication. Clin Transl Gastroenterol 2014;5:e66. doi:10.1038/ctg.2014.19

4. Rivkin A, Chagan L. Lubiprostone: Chloride channel activator for chronic constipation. Clin Ther 2006;28:2008-2021. doi:10.1016/j. clinthera.2006.12.013

5. Rao SS, Rattanakovit K, Patcharatrakul T. Diagnosis and management of chronic constipation in adults. Nat Rev Gastroenterol Hepatol 2016;13:295-305. doi:10.1038/nrgastro.2016.53

\section{Exertional heat stroke associated with acute liver failure in a young refugee. Implications in the era of social-economic crisis}

\section{To the Editor,}

Exertional heat stroke (EHS) is a life-threatening condition defined as the severe elevation of core body temperature and central nervous system dysfunction, which results from intensive exercise in hot or humid environment. It is a real medical emergency associated with multi-organ dysfunction. Acute liver failure (ALF) is a rare but potentially fatal late event in patients with EHS caused by systematic inflammatory activation [1]. We would like to report a case of EHS-related ALF in a young refugee.

The patient was a 27-year male of Albanian nationality, transferred to the University Hospital of Ioannina from the Greek Albanian border, in September 2016, due to confusion and an episode of loss of consciousness. He presented with hyperthermia, cognitive impairment, acute renal failure, rhabdomyolysis and hemodynamic instability. No previous medical record was able to be retrieved and the patient was 
not able to provide information due to impaired mental status. The patient received crystalloids and was transferred to the intensive care unit. Further deterioration of renal function and disseminated intravascular coagulation occurred within the following two days of admission and was treated with aggressive fluid administration, urine alkalization and fresh frozen plasma. Mental status initially improved but deteriorated again on the $3^{\text {rd }}$ day, when ALF was diagnosed. There was a progressive increase of aminotransferase levels (max value AST: $4834 \mathrm{IU} / \mathrm{ml}$, ALT: $6600 \mathrm{IU} / \mathrm{ml}$ ), of INR (3.5) and of total bilirubin $(12.8 \mathrm{mg} / \mathrm{dl})$. He was treated with $\mathrm{N}$-acetylcysteine (NAC) infusion at a dose of $100 \mathrm{mg} / \mathrm{kg} / 16$ hours, rifaximin and lactulose for 3 days. The patient's mental status gradually improved and was fully restored on the $5^{\text {th }}$ day, alongside the gradual normalization of the laboratory parameters. No other causes for ALF could be identified but physical exhaustion and hyperthermia and a late diagnosis of EHS was made. The patient confirmed that before collapsing, he had been walking for approximately 8 hours, covering about $40 \mathrm{~km}$ from Gjirokastër to Kakavia, mostly a rural area, in his effort to cross the border. The median ambient temperature that day was $28^{\circ} \mathrm{C}$ and the maximum was $37^{\circ} \mathrm{C}$, based on data from the weather station located at Ioannina. He had no past medical history and denied the use of any medicine.

Early recognition of EHS and the immediate external cooling of the body are essential to prevent organ failure and to improve outcome [2]. Rapid improvement of mental status during cooling period and the short interval between the high core temperature and cooling have been found to improve prognosis [3]. In our patient, external cooling was not applied due to the delayed diagnosis of EHS. Possible explanations for the positive outcome in our patient are the early transportation, in less than one hour, to the emergency department and the immediate support of organ functions.

Prognostic criteria and optimal management of EHSrelated ALF have not yet been elucidated. Metabolic acidosis, CPK $>1000$ IU/L, and elevated aminotransferases $>2$ UNL have been identified as predictors of EHS associated organ failure [4]. Serum phosphorus levels below $<0.5 \mathrm{mmol} / \mathrm{L}$ on admission are a predictive factor of ALF in patients with EHS [5]. All these parameters were present in our patient.

Although EHS has been mainly described in athletes and soldiers, increased awareness should exist for the occurrence of this medical emergency in special groups of people, such as refuges in the era of social economic crisis. Refuges are a vulnerable group of people, moving often under strenuous environmental conditions. Dealing with medical situations affecting refuges can be challenging as medical history information is lacking or the communication might be difficult. EHS-associated ALF should be suspected in refugees presented with hyperthermia and cognitive impairment who develop liver dysfunction. Early recognition of this rare complication could improve prognosis.

Dimitrios S. Politis ${ }^{1}$, Gerasimos Baltagiannis ${ }^{1}$, Nikolaos Tzampouras $^{1}$, George N. Kalambokis ${ }^{2}$, Vasileios Koulouras ${ }^{3}$, Dimitrios K. Christodoulou ${ }^{1}$

1) Department of Gastroenterology, University Hospital of Ioannina, School of Health Sciences, University of Ioannina; 2) $1^{\text {st }}$ Division of Internal Medicine, Medical School, University of Ioannina;. 3) Intensive care unit, University Hospital of Ioannina, Ioanin Ioannina, Greece

Correspondence: Dimitrios K. Christodoulou, dchristodoulou@gmail.com

Conflicts of interest: None.

DOI: $10.15403 /$ jgld-302

\section{REFERENCES}

1. Hart LE, Egier BP, Shimizu AG, Tandan PJ, Sutton JR. Exertional heat stroke: the runner's nemesis. Can Med Assoc J 1980;122:1144-1150.

2. Zeller L, Novack V, Barski L, Jotkowitz A, Almog Y. Exertional heatstroke: clinical characteristics, diagnostic and therapeutic considerations. Eur J Intern Med 2011;22:296-299. doi:10.1016/j. ejim.2010.12.013

3. Giercksky T, Boberg KM, Farstad IN, Halvorsen S, Schrumpf E. Severe liver failure in exertional heat stroke. Scand J Gastroenterol 1999;34:824827. doi:10.1080/003655299750025778

4. Varghese GM, John G, Thomas K, Abraham OC, Mathai D. Predictors of multi-organ dysfunction in heatstroke. Emerg Med J 2005;22:185-187. doi:10.1136/emj.2003.009365

5. Garcin JM, Bronstein JA, Cremades S, Courbin P, Cointet F. Acute liver failure is frequent during heat stroke. World J Gastroenterol 2008;14:158-159. doi:10.3748/wjg.14.158 